\title{
ZUM STUFENAUFBAU DES PARALLELENAXIOMS
}

Victor Pambuccian

EUCLID's parallel postulate is shown to be equivalent to the conjunction of the following two weaker postulates: "Any perpendicular to one side of a right angle intersects any perpendicular to the other side" and "For any acute angle $\angle O x y$, the segment $\mathrm{PQ}$ - where $\mathrm{P}$ is a point on $\mathrm{O} x, \mathrm{Q}$ a point on $\mathrm{O} y$ and $\mathrm{PQ} \perp \mathrm{O} y$ - grows indefinitely, i. e. can be made longer than any given segment".

\section{EINFÜHRUNG}

Wo immer wir in der Grundlegung der Geometrie den Tatbestand treffen, daß, sobald ein Teil $\mathcal{T}$ des Axiomensystems bereits vorhanden ist, ein Axiom $\alpha$ stärker ist als ein Axiom $\beta$, wobei weder $\alpha$ noch $\beta$ Sätze von $\mathcal{T}$ sind, stellt sich die Frage ob es eine geometrische Aussage $\beta^{\prime}$ gibt, die schwächer als $\alpha$ ist, aber zusammen mit $\beta$ dem Axiom $\alpha$ gleichwertig ist.

Vom logischen Standpunkt aus gibt es überhaupt kein Problem, denn was verlangt wird, ist: falls

$$
\mathcal{T} \vdash \alpha \rightarrow \beta, \mathcal{T} \not \forall \beta, \text { und } \mathcal{T}, \beta \not{ }^{\prime} \alpha \text {, }
$$

dann finde man $\beta^{\prime}$, so daß

$$
\mathcal{T} \vdash \alpha \leftrightarrow \beta \wedge \beta^{\prime} \text { und } \mathcal{T}, \beta^{\prime} \forall \alpha .
$$

Solch ein $\beta^{\prime}$ ist offensichtlich $\beta \rightarrow \alpha$. Die logische Formulierung des Problems läßt jedoch die Forderung, daß $\beta^{\prime}$ eine ,geometrische Aussage' sein soll, aus.

Unter einer ,geometrischen Aussage' ist eine solche zu verstehen, die man im Aufbau der Geometrie entweder als Axiom oder als Lehrsatz aufzustellen geneigt wäre. Wir wollen gleich zugeben, daß damit eigentlich nichts definiert wurde, und es ist auch nicht unsere Absicht die Gesamtheit der annehmbaren geometrischen Axiomen festzusetzen, denn damit würden wir uns ja anmaßen zu wissen welcher Form alle geometrisch bedeutsamen Aussagen sind. 
Da die meisten Axiomatisierungen der euklidischen Geometrie (s. z. B. [9]) al1s Allheits-ExistenzAussagen bestehen ( $\forall \exists$-Aussagen; d.h. es stehen alle Allquantoren $(\forall)$ vor allen Existenzquantoren $(\exists)$ ), wäre man geneigt (falls man die vorher erwähnten methodologischen Bedenken nicht billigt) die ,geometrische Aussage' als eine $\forall \exists$-Aussage in der logischen Sprache in der die Axiomatik verfaßt ist, aufzufassen. Dann würden aber einerseits Aussagen wie z.B. ,(Der Desarguessche Satz) oder (Es gibt ein Rechteck)', die offenbar als Axiome unerwünscht sind, für ,geometrische Aussagen' erklärt sein, und andererseits Aussagen wie z. B. die lokale Form des Parallelenaxioms, d. h. ,Es gibt einen Punkt $P$ und eine Gerade $g$, so daß es durch $P$ zu g höchstens eine Parallele gibt' nicht als ,geometrisch' anerkannt.

Das Problem der geometrisch bedeutungsvollen Aufspaltung von Axiomen wurde zum ersten Mal von Wanda SzMiELEW in [10] gelöst, wo sie eine geometrische Aussage $\beta^{\prime}$ aufgestellt hat (die besagt: ,Falls $A, B$ und $C$ drei verschiedene Punkte sind, dann schneidet die Mittelsenkrechte von $A B$ einen der Kreise die AC bzw. BC als Durchmesser haben'), für den Fall in dem $\mathcal{T}$ die PAsCh-freie ebene euklidische Geometrie ist, $\alpha$ das Kreisaxiom (,Eine Gerade, die durch das Innere eines Kreises geht, schneidet den Kreis') und $\beta$ das Axiom von PASCH ist.

Wir nennen ein solches $\beta^{\prime}$ kurz ein Bindeglied zwischen $\beta$ und $\alpha$ in bezug auf $\mathcal{T}$ und sagen, da $\beta \alpha$ in bezug auf $\mathcal{T}$ in $\beta$ und $\beta^{\prime}$ aufgespalten wurde.

Diese Aufspaltung von Axiomen ist deshalb sinnvoll, weil sie uns eine bessere Übersicht des Stufenaufbaus der betreffenden Theorie gestattet. Denn eine endlich-axiomatisierbare Theorie könnte eigentlich auch durch ein einziges Axiom axiomatisiert werden, für das man die Konjunktion aller Axiome eines ihrer endlichen Axiomensysteme wählen könnte. Der Nachteil wäre, daß dann die ganze Theorie in einem Zug aufgebaut würde. Die Axiomatisierungen mit mehreren Axiomen können somit bereits als Aufspaltungen des einen Axioms aufgefaßt werden und zeugen vom Interesse, daß die mathematische Praxis an Aufspaltungen hat.

Ein weiterer Grund warum Aufspaltungen wichtig sind, ist der, daßs sie zu Axiomensystemen führen, die dem Ideal eines einfachen Axiomensystems näherkommen, wobei wir unter ,einfach' ein solches Axiomensystem verstehen, bei dem kein Axiom einer nicht-trivialen Aufspaltung fähig ist. ${ }^{1}$

Ist $\Sigma$ ein endliches Axiomensystem und $\alpha$ ein Axiom aus $\Sigma$, so wird $\alpha$ aufspaltbar genannt, falls es Aussagen $\beta$ und $\gamma$ gibt, so daß

$$
\Sigma \backslash\{\alpha\} \vdash \alpha \leftrightarrow \beta \wedge \gamma \text { und } \Sigma \backslash\{\alpha\} \not \beta \rightarrow \alpha, \Sigma \backslash\{\alpha\} \not \gamma \rightarrow \alpha .
$$

Es gibt aber Aufspaltungen von $\alpha$ bei denen man die Axiome $\beta$ und $\gamma$ nicht für Vereinfachungen von $\alpha$ halten kann; es ist nämlich oft möglich $\alpha$ in $\alpha \vee \delta$ und $\alpha \vee \neg \delta$ aufzuspalten, wobei $\delta$ eine Aussage ist.

\footnotetext{
${ }^{1}$ Dieses Einfachheitskriterium für endliche Axiomensysteme stammt von O. Helmer [4], und wurde in [7] so verändert, daß die Aufspaltung von $\forall a b a b \equiv b a$ in $\forall a a a \equiv a a$ und $\forall a b \neg(a=b) \rightarrow a b \equiv b a$ auch als trivial gilt. Da wir die in [7] vorgeschlagene Veränderung des HELMERschen Einfachheitskriteriums inzwischen für ungeeignet halten (da zu viele Aufspaltungen dadurch ausgeschlossen werden), kehren wir zur Helmerschen Definition der , trivialen' Aufspaltung zurück, und lassen somit Aufspaltungen der oben angegebenen Art als ,nicht-trivial' zu.
} 
Als geometrisches Bejspiel wäre folgendes zu nennen: $\alpha$ ist das Axiom ,Es gibt ein Rechteck $(R)$ ', $\beta$ und $\gamma$ sind ,Die Mittellinie in einem Dreieck ist $\leq$ als die Hälfte der ihr gegenüberliegenden Seite' bzw. ,Die Mittellinie in einem Dreieck ist $\geq$ als die Hälfte der ihr gegenüberliegenden Seite'; $R$ besagt, daß die metrische Konstante (s. $\{2) k=0$ ist ; $\beta$, daß $k \leq 0$ ist und $\gamma$, daß $k \geq 0$ ist.

Wir werden derartige Aufspaltungen des Axioms $\alpha$ (nämlich als $\alpha \vee \delta$ und $\alpha \vee \neg \delta$ ), auch wenn sie zwei ,geometrische Aussagen' $\beta$ und $\gamma$ produzieren, als trivial bezeichnen, und nur nicht-triviale Aufspaltungen suchen.

M. DEHN hat in [2] Beispiele nicht-archimedischer Ebenen mit euklidischer Metrik gegeben, in denen das euklidische Parallelenaxiom verletzt wird, womit er bewiesen hat, daß das Axiom $R$ in bezug auf die absolute Geometrie $(\mathcal{A})^{2}$ schwächer ist als das (Playfairsche) euklidische Parallelenaxiom (Par). F. BACHMANN hat in [1] eine weitere Abschwächung des Axioms $R$ vorgeschlagen, nämlich, Jedes Vierseit mit drei Rechten Winkeln schließt sich (A)' (s. Figur 1$)^{3}$

Es wird in [1] bewiesen, daß in $\mathcal{A}$ die Implikations-Reihe

$$
\operatorname{Par} \rightarrow R \rightarrow A, \text { mit } A \nrightarrow R \nrightarrow P \text { ar gilt. }
$$

Es stellt sich somit das Problem, zwei Bindeglieder, $\beta_{1}$ und $\beta_{2}, \beta_{1}$ von $A$ zu $R$ und $\beta_{2}$ von $R$ zu $P a r$ zu finden, so daß $\mathcal{A} \forall A \wedge \beta_{2} \rightarrow P a r$, und somit das euklidische Parallelenaxiom in drei Axiome, $A, \beta_{1}$ und $\beta_{2}$ aufzuspalten. Dieses Problem bleibt offen.

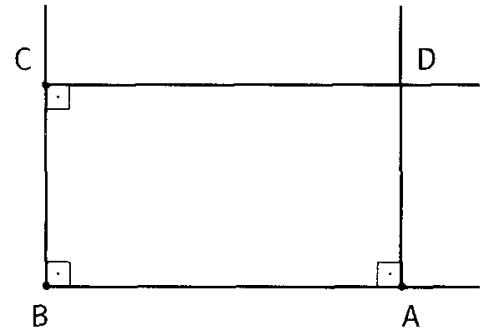

Fig.1

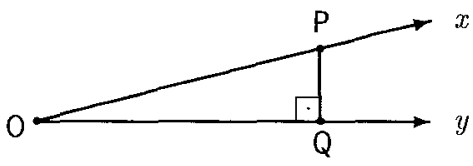

Fig.2

\footnotetext{
${ }^{2}$ Die ebene absolute Geometrie ist hier als diejenige Theorie verstanden, die durch die Axjome $A 1-A 9$ aus [9], oder durch die Axiome I 1-3, II, III aus [6] gegeben wird.

${ }^{3}$ In der logischen Sprache erster Ordnung $L_{B D}$-wobei , $B(a b c)^{\prime}$ als , $a, b, c$ sind drei kollineare Punkte und $b$ liegt zwischen $a$ und $c^{\prime}$, und, $D(a b c d)^{\prime}$ als, die Strecke $a b$ ist zur Strecke $c d$ kongruent'zu lesen sind (s. auch [9]) - könnte man $A$ folgendermaßen ausdrücken:

$\forall a b c x y z t u \exists d \neg(a=b) \wedge \neg(b=c) \wedge B(x b a) \wedge b a \equiv b x \wedge B(y c b) \wedge c b \equiv c y \wedge z b \equiv z y \wedge \neg(z=c)$

$\wedge B(t a b) \wedge a t \equiv a b \wedge \neg(u=a) \wedge u b \equiv u t \rightarrow L(c z d) \wedge L(a u d)$,

wobei, $L(a b c)^{\prime}$ eine Abkürzung von $B(a b c) \vee B(b c a) \vee B(c a b)$ ist und , $a b \equiv c d$ ' eine leichter lesbare Schreibweise für, $D(a b c d)^{\prime}$ 'ist.

F. BACHMANN hat in $[1,5.176]$ gezeigt, daß das Axiom $A$ (das , Lotschnittaxiom') zu folgender Spezialisierung des euklidischen Parallelenaxioms (das man auch, wie z. B. im Axiomensystem aus [9], als, Durch jeden Punkt im Innern eines Winkels gibt es eine Gerade, welche beide Schenkel trifft' ausdrücken kann) äquivalent ist: ,Durch jeden Punkt im Inneren eines rechten Winkels gibt es eine Gerade, welche beide Schenkel trifft'.
} 
Wir werden in dieser Note ein Bindeglied zwischen $A$ und $P a r$ in bezug auf $\mathcal{A}$ finden. Das Bindeglied ist nämlich das ,ARIstotelische Axiom' $(A r)$, dessen Tragweite, mit besonderer Berücksichtigung der Bedeutung von Ar für die Grundlegung der hyperbolischen Geometrie, von M. J. Greenberg in [3] untersucht wurde.

Es besagt (s. Figur 2), daß die Längen der Strecken, deren Endpunkte auf den Schenkeln eines gegebenen spitzen Winkels liegen und die senkrecht auf einem der Schenkel sind, unbegrenzt wachsen, d.h. länger als jede gegebene Strecke gemacht werden können ${ }^{4}$.

\section{Algebraische Beschreibung der H-Ebenen in denen das Lot- SCHNITTAXIOM GILT}

Da wir synthetisch nicht beweisen konnten, daß

$$
\mathcal{A} \vdash A \wedge A r \rightarrow P a r
$$

werden wir die PEJAssche (s. [8], [5], [1]) algebraische Beschreibung der Modelle der ebenen absoluten Geometrie (die wir im folgenden H-Ebenen nennen werden) benützen müssen.

Wir werden hier kurz die Resultate aus [1] zusammenfassen.

Sei $K$ ein Körper und $k$ ein Element aus $K$. Unter einer affin-metrischen Ebene $\mathbf{A}(K, k)$ (s. $[5$, S.215] ) über dem Körper $K$ mit der metrischen Konstanten $k$ verstehen wir die projektive Koordinatenebene $\mathbf{P}(K)$ über $K$, für deren Punkte der Gestalt $(x, y, 1)$ wir kurz $(x, y)$ schreiben, in der die Geraden $[u, v, w]$ und $\left[u^{\prime}, v^{\prime}, w^{\prime}\right]$ orthogonal sind genau dann wenn

$$
u u^{\prime}+v v^{\prime}+k w w^{\prime}=0 .
$$

Ist der Körper $K$ angeordnet, so kann man den affinen Teil $\mathbf{A}(K)$, der aus allen Punkten von $\mathbf{P}(K)$ besteht, die nicht auf der Geraden $[0,0,1]$ liegen, wie üblich anordnen.

Die algebraische Beschreibung der H-Ebenen besteht aus der Angabe einer Punktmenge $E$ einer affin-metrischen Ebene $\mathbf{A}(K, k)$, die die Punktmenge der H-Ebene ist. Da $E$ stets in $\mathbf{A}(K)$ liegen wird, übernimmt die H-Ebene die Anordnung von $\mathbf{A}(K)$. Die Kongruenz zweier Strecken ab und cd wird, falls $E \subset \mathbf{A}(K, 0)$ ist, durch das Bestehen der Gleichung

$$
\left(a_{1}-b_{1}\right)^{2}+\left(a_{2}-b_{2}\right)^{2}=\left(c_{1}-d_{1}\right)^{2}+\left(c_{2}-d_{2}\right)^{2}
$$

und, falls $E \subset \mathbf{A}(K, k)$ mit $k \neq 0$, durch das Bestehen der Gleichung

$$
\frac{F(\mathbf{a}, \mathbf{b})^{2}}{Q(\mathbf{a}) Q(\mathbf{b})}=\frac{F(\mathbf{c}, \mathbf{d})^{2}}{Q(\mathbf{c}) Q(\mathbf{d})}
$$

\footnotetext{
${ }^{4} \forall o x y y^{\prime} a \exists p q q^{\prime} z \quad B\left(y^{\prime} o y\right) \wedge o y \equiv o y^{\prime} \wedge \neg(a=o) \wedge a y^{\prime} \equiv a y \wedge B(a x y) \rightarrow(B(o x p) \vee B(o p x))$ $\wedge(B(o y q) \vee B(o q y)) \wedge B\left(o q q^{\prime}\right) \wedge q o \equiv q q^{\prime} \wedge p o \equiv p q^{\prime} \wedge p q \equiv o z \wedge B(o y z)$.
} 
gegeben, wobei

$$
F(\mathbf{x}, \mathbf{y})=k\left(x_{1} y_{1}+x_{2} y_{2}\right)+1, Q(\mathbf{x})=F(\mathbf{x}, \mathbf{x}), \text { und } \mathbf{x}=\left(x_{1}, x_{2}\right), \mathbf{y}=\left(y_{1}, y_{2}\right)
$$

Von nun an sei $K$ ein angeordneter pythagoreischer Körper, $R$ der Ring der ganzzahlig einschließbaren Elemente von $K$, d.h. $R=\{x \in K|(\exists n \in \mathrm{N})| x \mid<n\}$ und $P$ das Ideal der unendlich kleinen Elemente aus $K$, d. h. $P=\{0\} \cup\left\{x \in K \mid x^{-1} \notin R\right\}$.

F. BaCHMANn zeigt in [1], daßs die H-Ebenen, in denen das Axiom $A$ gilt, von zwei Typen sind, nämlich

Typ I (entspricht dem Theorem 1 aus [1])

$E=\{(a, b) \mid a, b \in M\} \subset \mathbf{A}(K, 0)$, wobei $M$ ein $R-M o d u l \neq(0)$ ist;

Typ II (entspricht dem Theorem 2 aus [1])

$E=\{(a, b) \mid a, b \in M\} \subset \mathbf{A}(K, k)$ mit $k \neq 0$, wobe $i M$ ein in

$\left\{a \in K \mid k a^{2} \in P\right\}$ eingeschlossener $R$-Modul $\neq(0)$ ist, der die Bedingung

$$
a \in M \Rightarrow k a^{2}+1 \in K^{2}
$$

erfüllt $\left(K^{2}=\left\{a^{2} \mid a \in K\right\}\right)$.

\section{D AS ARISTOTELISCHE AXIOM GILT NICHT IN H-EBENEN VOM TYP II}

Wir werden nun beweisen, daß

$$
\mathcal{A} \vdash A \wedge A r \rightarrow R .
$$

M.J. Greenberg ([3, Theorem 1]) hat bewiesen, daß in einer H-Ebene, in der Argilt, die metrische Konstante $k \leq 0$ ist. H-Ebenen, in denen sowohl $A$ als auch $A r$ gilt, können demnach nur vom Typ I oder vom Typ II mit $k<0$ sein. Wir werden den folgenden Satz beweisen:

SATZ 1 In H-Ebenen vom Typ II mit $k<0$ gilt Ar nicht.

Beweis. ${ }^{5}$ Da die metrische Konstante $k$ nur bis auf einen quadratischen Faktor $\neq 0$ durch die gegebene H-Ebene festgelegt ist, können wir o. B. d. A. annehmen, daß $k \in K \backslash P$ ist.

Da $M \subset\left\{a \in K \mid k a^{2} \in P\right\}$ wird $M \subseteq P$ sein. Insbesondere wird $a b \in M$ falls $a, b \in M$, da $M$ ein $R$-Modul ist und $M \subseteq P \subset R$ ist.

Es sei $p>0$ ein Element aus $M$, und $\overrightarrow{O x}$ bzw. $\overrightarrow{O y}$ (wobei $\mathrm{O}=(0,0)$ ist) die Halbgeraden in $\mathbf{A}(K, k)$, deren Punkte die Koordinaten $(m, p m)$ bzw. $(m, 0)$, mit $m \in M, m>0$ haben $(O=(0,0))$. Wir

\footnotetext{
${ }^{5}$ Ich möchte dem Gutachter an dieser Stelle für die Vereinfachung dieses Beweises danken.
} 
behaupten, daß jede Strecke deren Endpunkte $\mathrm{P}$ und $\mathrm{Q}$ auf $\overrightarrow{\mathrm{O} x}$ bzw. $\overrightarrow{\mathrm{O} y}$ so liegen, daß $\mathrm{PQ} \perp \overrightarrow{\mathrm{O} y}$, kürzer als die Strecke $\mathrm{OB}$ sein wird, wobei $\mathrm{B}=(p, 0)$.

Ist $\mathrm{P}=(m, p m)$, so muß $\mathrm{Q}=(m, 0)$ sein, da $\mathrm{PQ}$ zu $\overrightarrow{\mathrm{O} y}$ orthogonal ist. Wir tragen die Strecke $\mathrm{PQ}$ aus $O$ auf $\overrightarrow{O x}$ ab, und erhalten dadurch einen Punkt $C=(c, 0)$, so daß $O C$ und $P Q$ kongruent sind, d.h., laut (3),

$$
\frac{F((m, p m),(m, 0))^{2}}{Q((m, p m)) Q((m, 0))}=\frac{F((0,0),(c, 0))^{2}}{Q((0,0)) Q((c, 0))}
$$

Aus (5) erhalten wir

$$
c^{2}=\frac{p^{2} m^{2}}{1+k m^{2}}
$$

und somit ist $0<c<p$, da $m^{2}$ und $k m^{2}$, und folglich auch $\frac{m^{2}}{1+k m^{2}}$, unendlich klein sind. Somit liegt $\dot{C}$ stets zwischen $\mathrm{O}$ und $\mathrm{B}$.

Folglich können H-Ebenen, in denen sowohl $A$ als auch Ar gilt, nur vom Typ I sein, und das sind gerade diejenigen H-Ebenen, in denen $R$ gilt (s. [8] oder [1]). Damit ist (4) bewiesen.

\section{H-EBEnEN MIT EUKLIDISChER METRIK IN DENEN DAS ARISTOTE- LISCHE AXIOM GILT}

Wir werden nun beweisen, daß

$$
\mathcal{A} \vdash R \wedge A r \rightarrow \text { Par. }
$$

Es sei g eine Gerade und 0 ein Punkt der nicht auf $g$ liegt (s. Figur 3). Wir wollen beweisen, daß es durch $\mathrm{O}$ zu g eine einzige Parallele gibt.

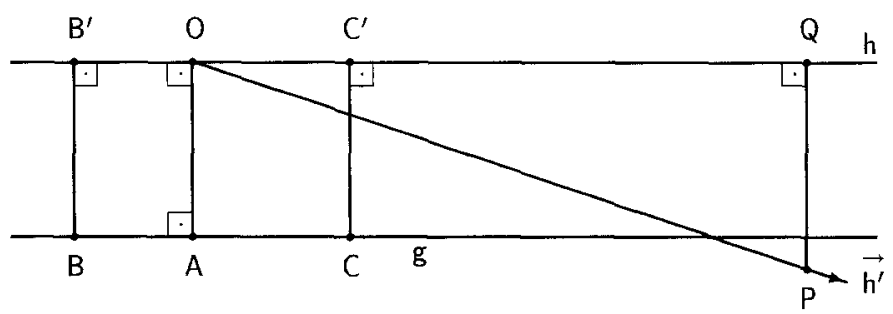

Fig. 3

Es sei $A$ der Fußpunkt der Senkrechten von $O$ auf $g$, und es sei $h$ die Senkrechte in $O$ auf OA. Es seien $B$ und $C$ zwei Punkte auf $g$, so daß $A$ zwischen $B$ und $C$ liegt, und es seien $B^{\prime}$ und $C^{\prime}$ die Fußpunkte der Senkrechten von B bzw. $C$ auf $h$. Es sei nun $h^{\prime}$ eine von $h$ verschiedene Gerade, die durch $O$ geht. Wir wollen beweisen, daß sich $h^{\prime}$ und $g$ schneiden. $h^{\prime}$ muß (den Anordnungsaxiomen und insbesondere dem Axiom von PASCH zufolge) entweder durch das Innere des Rechtecks OACC 
oder durch das Innere des Rechtecks $\mathrm{OABB}^{\prime}$ gehen. O. B. d. A. können-wir annehmen, daß $\mathrm{h}^{\prime}$ durch das Innere des Rechtecks OACC' geht. Es sei $\overrightarrow{h^{\prime}}$ diejenige Halbgerade von $h^{\prime}$, die von 0 ausgeht und durch das Innere von $O A C C^{\prime}$ geht. Ist $h^{\prime}$ von der Geraden $O A$ verschieden, so ist der von den Halbgeraden $\overrightarrow{h^{\prime}}$ und $\overrightarrow{O C^{\prime}}$ gebildete Winkel spitz, und folglich gibt es, laut Ar, zwei Punkte $\mathrm{P}$ und $Q$ auf $\overrightarrow{h^{\prime}}$ bzw. $\overrightarrow{O C^{\prime}}$, so daß $P Q \perp \overrightarrow{O C^{\prime}}$ ist und $P Q$ länger als $O A$ ist. Da der Abstand zwischen $h$ und $g$ überall derselbe ist, müssen die Punkte $P$ und $Q$ auf verschiedenen Seiten der Geraden $g$ liegen, und somit liegen auch $O$ und $P$ auf verschiedenen Seiten der Geraden g. Dem Axiom von PASCH zufolge, schneidet OP (d.h. $\overrightarrow{h^{\prime}}$ ) die Gerade g, w. z. b. w.

\section{$5\{A, A r\}$ ist eine nicht-triviale Aufspaltung von Par}

Aus (4) und (6) folgt, daß

$$
\mathcal{A} \vdash A \wedge A r \rightarrow P a r \text {. }
$$

Da $\mathcal{A} \vdash P$ ar $\rightarrow A \wedge A r$ wohlbekannt ist, $\mathcal{A} \forall \forall \rightarrow P$ ar in [1] (s. (1)), und $\mathcal{A} \not \forall A r \rightarrow P$ ar in $[3$, Theorem 2] (Ar gilt auch in der hyperbolischen ebenen Geometrie) bewiesen wurde, ist $\{A, A r\}$ eine Aufspaltung von $P$ ar in bezug auf $\mathcal{A}$.

Wäre nun $\{A, A r\}$ eine triviale Aufspaltung von $P a r$, dann würde es eine Aussage $\delta$ geben, so daß $\mathcal{A} \vdash A \leftrightarrow \operatorname{Par} \vee \delta$ und $\mathcal{A} \vdash A r \leftrightarrow \operatorname{Par} \vee \neg \delta$, und dann müßte auch $\mathcal{A} \vdash A \vee A r$, was falsch ist, da es, wie wir im folgenden zeigen werden, H-Ebenen gibt, in denen weder $A$ noch Ar gilt.

Die H-Ebenen, in denen $A$ nicht gilt sind vom

Typ III (entspricht dem Theorem 3 aus [1])

$E=\{\mathbf{x} \mid Q(\mathbf{x})>0, Q(\mathbf{x}) \notin J\} \subset \mathbf{A}(K, k)$ mit $k<0$, wobei $J \subseteq P$ ein Primideal von $R$ ist, der die Bedingung

$$
k a^{2}+1>0, k a^{2}+1 \notin J \Rightarrow k a^{2}+1 \in K^{2}
$$

erfüllt, und wobei $K$ die Bedingung

$$
\left\{a \in K \mid k a^{2} \in R \backslash P\right\} \neq \emptyset
$$

erfüllt.

SATZ 2 Es sei $K$ ein nichtarchimedischer euklidischer angeordneter Körper, $k=-1$ und $J=P$. Dann gilt in der entsprechenden H-Ebene vom Typ III weder A noch Ar.

Beweis. Es sei $p \in P$ und $\overrightarrow{\mathrm{O} x}, \overrightarrow{\mathrm{O} y}$ wie in Satz 1 , mit dem Unterschied, daB nun $m \in K$ und die Punkte auf diesen Halbgeraden aus der Menge $E$ des Typs III sein muß, d. h. für einen Punkt $(m, 0)$ auf $\overrightarrow{\mathrm{O} y}$ muß

$$
1-m^{2}>0 \text { und } 1-m^{2} \notin P \text { sein. }
$$


Wir behaupten, daß jede Strecke, deren Endpunkte $P$ und $Q$ auf $\overrightarrow{O x}$ bzw. $\overrightarrow{O_{y}}$ so liegen, daß $P Q \perp \overrightarrow{O y}$, kürzer als die Strecke $O B$ sein wird, wobei

$$
\mathrm{B}=\left(\frac{1}{2}, 0\right) \text {. }
$$

Es sei $P \in \overrightarrow{\mathrm{O} x}, Q \in \overrightarrow{\mathrm{O} y}, \mathrm{PQ} \perp \overrightarrow{\mathrm{O} y}$ und $\mathrm{C}=(c, 0)$ auf $\overrightarrow{\mathrm{O} y}$, so da $\beta \mathrm{OC}$ kongruent zu $\mathrm{PQ}$ ist. Dann gilt, wie in Satz 1

$$
c^{2}=\frac{p^{2} m^{2}}{1-m^{2}}
$$

Da, laut (7), $m^{2}<1$ und $\left(1-m^{2}\right)^{-1} \in R$ ist, und $p$ in $P$ gewählt wurde, muß $c^{2}$ und somit auch $c$ in $P$ und folglich kleiner als $\frac{1}{2}$ sein. Somit ist die Strecke $O C$, und folglich auch $P Q$, kleiner als $\mathrm{OB}$.

Satz 2 folgt auch aus [3, Theorem 3].

\section{Das lotschnittaxiom als Allheits-Aussage}

F. Bachmann bewies in $[1$, Satz 6$]$, daß das Axiom $A$ erblich ist, d.h. gilt $A$ in einer $H$-Ebene $\mathbf{H}$, so gilt $A$ in jeder $\mathbf{H}$-Ebene $\mathbf{H}^{\prime} \subset \mathbf{H}$.

Vom logischen Standpunkt aus gesehen, ist As Erblichsein gleichbedeutend mit der Existenz einer Allheitsaussage $A^{\prime}$, die mit $A$ äquivalent ist d.h., so daß

$$
\mathcal{A} \vdash A \leftrightarrow A^{\prime}
$$

Obwohl in [1] drei Äquivalente des Axioms $A$ angegeben werden, ist keines davon eine Allheitsaussage. Wir werden im folgenden zeigen, daß

\section{SATZ 3 Die Allheitsaussage}

$A^{\prime} . \forall p p^{\prime} o o^{\prime} m m^{\prime} q r \neg(q=o) \wedge \neg\left(m=m^{\prime}\right) \wedge B\left(m^{\prime} o m\right) \wedge m o \equiv m o^{\prime} \wedge m^{\prime} o \equiv m o \wedge B\left(o m o^{\prime}\right)$

$\wedge q m \equiv q m^{\prime} \wedge p o \equiv p o^{\prime} \wedge p o \equiv p r \wedge q o \equiv q r \wedge B(o q r) \wedge p q \equiv p m \wedge p m \equiv o p^{\prime}$

$\wedge\left(B\left(o p^{\prime} o^{\prime}\right) \vee B\left(o o^{\prime} p^{\prime}\right)\right) \rightarrow B\left(o p^{\prime} o^{\prime}\right) \wedge \neg\left(p^{\prime}=o^{\prime}\right)$

ist mit $A$ äquivalent.

$A^{\prime}$ besagt, daß in einem gleichschenkligen Dreieck, dessen Basiswinkel halbrechtwinklig sind, die Basishöhe kleiner als die Basis ist (s. Figur 4).

Beweis. Um (8) zu beweisen, gen ügt es zu zeigen, daß $A^{\prime}$ in den Ebenen vom Typ I und II aber nicht in den Ebenen vom Typ III gilt. $A^{\prime}$ gilt offenbar in H-Ebenen vom Typ I, da in ihnen die Basishöhe im Dreieck $\triangle \mathrm{POO}^{\prime}$ gleich der Hälfte der Basis ist. 
Für H-Ebenen vom Typ II und III sei $\mathrm{O}=(0,0), 0 \mathrm{O}^{\prime}$ die $x$-Achse und $\mathrm{P}=(m, m)$ mit $m>0$. Dann ist $\mathrm{M}=(m, 0), \mathrm{O}^{\prime}=\left(\frac{2 m}{1-k m^{2}}, 0\right)$ und $\mathrm{P}^{\prime}=\left(\frac{m}{\sqrt{1+k m^{2}}}, 0\right)\left(\sqrt{1+k m^{2}}\right.$ ist hier die positive Lösung der Gleichung $\left.X^{2}=1+k m^{2}\right)$.

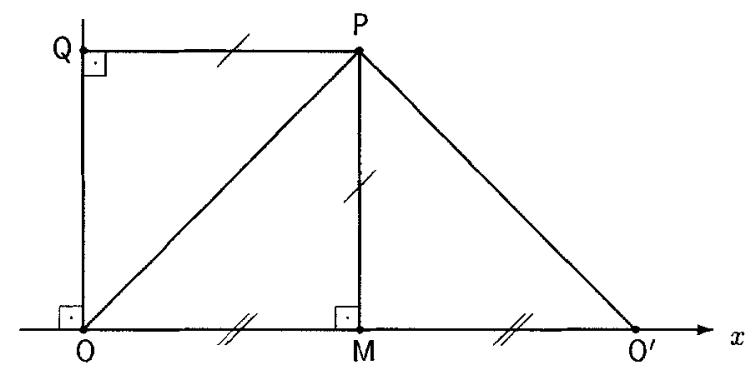

Fig. 4

Wir werden beweisen, daß die Ungleichung dessen Bestehen vom Axiom $A^{\prime}$ gefordert wird, nämlich

$$
\frac{m}{\sqrt{1+k m^{2}}} \leq \frac{2 m}{1-k m^{2}}
$$

in allen Ebenen vom Typ II gültig ist, daß es aber in allen Ebenen vom Typ III Punkte $P=(m, m)$ gibt für die (9) nicht besteht. Man rechnet leicht nach, daß (9) mit

$$
\left(k m^{2}\right)^{2}-6 k m^{2} \leq 3
$$

gleichbedeutend ist.

In H-Ebenen vom Typ II ist die linke Seite der Ungleichung (10) unendlich klein, da $M \subset\{a \in K \mid$ $\left.k a^{2} \in P\right\}$ ist, und somit ist $(10)$ in diesen H-Ebenen gültig.

In H-Ebenen vom Typ III muß, damit $\mathrm{P}=(m, m)$ der Menge $E$ angehört

$$
2 k m^{2}+1>0 \text { und } 2 k m^{2}+1 \notin J \text { sein. }
$$

Sei $x=k m^{2}$. Dann ist $(10)$ gleichbedeutend mit

$$
3-2 \sqrt{3} \leq x \leq 3+2 \sqrt{3}
$$

und (11) wird sicher erfüllt sein falls $x>-\frac{1}{2}$ und $x+\frac{1}{2} \notin P$. Da $\left\{a \in K \mid k a^{2} \in R \backslash P\right\} \neq \emptyset$ ist, sei $a_{0} \in K$, so daß $k a_{0}^{2} \in R \backslash P$. Es gibt somit zwei natürliche Zahlen $p$ und $q$, so daßs $-\frac{1}{2}+\frac{1}{100}<k\left(\frac{p}{q} a_{0}\right)^{2}<3-2 \sqrt{3}$. Wählen wir nun $\mathrm{P}=(m, m)$ mit $m=\frac{p}{q} a_{0}$, so wird $\mathrm{P}$ in $E$ sein, und (10), und somit auch (9), wird nicht erfüllt sein, d.h. im Dreieck $\triangle P O O^{\prime}$ ist die Höhe aus $P$ länger als $0 \mathrm{O}^{\prime}$.

Herrn Prof. Dr. Andreas Blass bin ich für viele Gespräche, die mir zur Klärung des Aufspaltungsproblems hilfreich waren, zu Dank verpflichtet. 


\section{LITERATUR}

[1] F. Bachmann, Zur Parallelenfrage, Abh. Math. Sem. Univ. Hamburg 27 (1964), 173-192.

[2] M. DehN, Die LegendRE'schen Sätze über die Winkelsumme im Dreieck, Math. Ann. 53 (1900), 404-439.

[3] M. J. Greenberg, Aristotle's axiom in the foundations of geometry, J. of Geometry 33 (1988), 53-57.

[4] O. HeLmer, A few remarks on the syntax of axiom-systems, Actes du Congrès International de Philosophie Scientifique (Paris, 1935), Vol. VII, Hermann, Paris, 1936, p. 12-17.

[5] G. Hessenberg, J. Diller, Grundlagen der Geometrie, W. de Gruyter, Berlin, 1967.

[6] D. HilberT, Grundlagen der Geometrie, 12. Auflage, B. G. Teubner, Stuttgart, 1977.

[7] V. Pambuccian, Simplicity, Notre Dame J. Formal Logic 29 (1988), 396-411.

[8] W. Pejas, Die Modelle des Hilbertschen Axiomensystems der absoluten Geometrie, Math. Ann. 143 (1961), 212-235.

[9] W. SchwabhäUser, W. Szmielew, A. Tarski, Metamathematische Methoden in der Geometrie, Springer-Verlag, Berlin, 1983.

[10] W. SZMIELEW, A statement on two circles as the geometric analog to EuCLiD's field property, Bull. Acad. Polon. Sci. Sér. Sci. Math., Astronom., Phys. 18 (1970), 759-764.

Department of Mathematics

University of Michigan

Ann Arbor, MI 48109-1003

U.S.A.

Eingegangen am 23. März 1992 